\title{
Diluted Magnetic Semiconductors InFeSb Prepared by Laser Ablation: Spectroscopic and Microscopic Investigations
}

\author{
Elena Gan'shina ${ }^{1}$, Leonard Golik ${ }^{2}$, Zoya Kun'kova ${ }^{2}$, Georgy Zykov ${ }^{1, *}$, Alexander Rukovishnikov², Marina Temiryazeva ${ }^{2}$, \\ Vladimir Lesnikov ${ }^{3}$, Yuri Markin ${ }^{2}$ \\ ${ }^{1}$ Lomonosov Moscow State University, Department of Physics, 119991 Moscow Leninskie Gory, Russia \\ 2RAS, Kotel'nikov Institute of Radioengineering and Electronics (Fryazino Branch), 141190 Fryazino, Russia \\ ${ }^{3}$ RAS, Lobachevsky Institute of Physics and Technology, 603950 Nizhny Novgorod, Russia
}

\begin{abstract}
We report optical and magneto-optical results as well as atomic force microscopy (AFM) and magnetic force microscopy (MFM) results for InFeSb samples prepared by laser ablation. AFM and MFM studies have revealed the presence of magnetic particles on the samples surface, whose sizes depend on the Fe content and substrate temperature. It has found that both optical and magneto-optical spectra are superposition of spectra from the doped InFeSb layers and particles on their surface.
\end{abstract}

\section{Introduction}

Spintronic devices suggested in theoretical studies may possess characteristics that are unreachable for traditional electronics. However, the implementation of such devices is restrained by the lack of ferromagnetic semiconducting (FMS) thin films, whose Curie temperature (Tc) is above room one. Recently, sets of FMS GaFeSb [1] and InFeSb [2] thin films obtained using molecular beam epitaxy and laser ablation with $\mathrm{T}_{\mathrm{C}}$ higher than $300 \mathrm{~K}$ have been reported. Magneto-optical spectroscopy is a powerful tool for probing the electronic structures of diluted magnetic semiconductors (DMS). It can provide unique information about such systems because MOKE spectra are sensitive to electronic and magnetic properties. We can distinguish features in the MO spectra associated with transitions in a doped semiconductor matrix (intrinsic ferromagnetism) and features associated with the presence of magnetic inclusions, since they are observed at different energies. In this work we study InFeSb thin films, which have been prepared by laser ablation [2], using optical (ellipsometry) and magneto-optical (transversal Kerr effect, TKE) spectroscopy as well as atomic and magnetic force microscopy (AFM and MFM, respectively).

\subsection{Samples and experimental specifications}

\subsubsection{Samples}

InFeSb samples are $\approx 40 \mathrm{~nm}$-thick films, which were fabricated on i-GaAs substrates by pulsed laser deposition. Solid InSb- and Fe-targets were periodically sputtered by Nd:YAG laser in a vacuum chamber. The ratio of the sputtering times, $\mathrm{t}_{\mathrm{Fe}} /\left(\mathrm{t}_{\mathrm{InSb}}+\mathrm{t}_{\mathrm{Fe}}\right)\left(\mathrm{Y}_{\mathrm{Fe}}\right)$, was varied in the range of $0.08-0.17$. The substrate temperature, $\mathrm{T}_{\mathrm{S}}$, was $20,150,200,250^{\circ} \mathrm{C}$ and $300^{\circ} \mathrm{C}$. The samples characteristics are displayed in Table 1.

Table 1. InFeSb samples sputtered on GaAs substrate.

\begin{tabular}{|c|c|c|}
\hline $\begin{array}{c}\text { Sample } \\
\text { number }\end{array}$ & $\begin{array}{c}\text { Technology } \\
\text { parameter, } \mathrm{Y}_{\mathrm{Fe}}\end{array}$ & $\begin{array}{c}\text { Substrate } \\
\text { temperature, } \\
\mathbf{T}_{\mathbf{s}},{ }^{\circ} \mathbf{C}\end{array}$ \\
\hline $\mathrm{N} 1$ & 0.08 & 250 \\
\hline $\mathrm{N} 2$ & 0.12 & 20 \\
\hline $\mathrm{N} 3$ & 0.12 & 150 \\
\hline $\mathrm{N} 4$ & 0.12 & 200 \\
\hline $\mathrm{N} 5$ & 0.12 & 250 \\
\hline $\mathrm{N} 6$ & 0.17 & 250 \\
\hline $\mathrm{N} 7$ & 0.17 & 300 \\
\hline
\end{tabular}

\subsubsection{Experimental methods}

Magneto-optical properties were studied in the transversal Kerr effect (TKE) geometry. TKE is an intensity change of reflected linear p-polarized light during a sample magnetization. Optical properties were measured using spectral ellipsometry. The TKE measurement parameters were $\mathrm{E}=0.5-4.0 \mathrm{eV}, \mathrm{T}=20-$ $460 \mathrm{~K} ; \mathrm{H}$ up to $3 \mathrm{kOe}$. Ellipsometry spectra were

\footnotetext{
* Corresponding author: V0tum-Separatum@yandex.ru
} 
obtained in the energy range of $\mathrm{E}=1.2-4.4 \mathrm{eV}$ at room temperature.

\section{Results}

The AFM and MFM images (Fig. 1, left and right sides, respectively) obtained at room temperature show complex surface topography of the samples. There are particles, whose sizes depend on the Fe quantity and substrate temperature, on the samples surface.

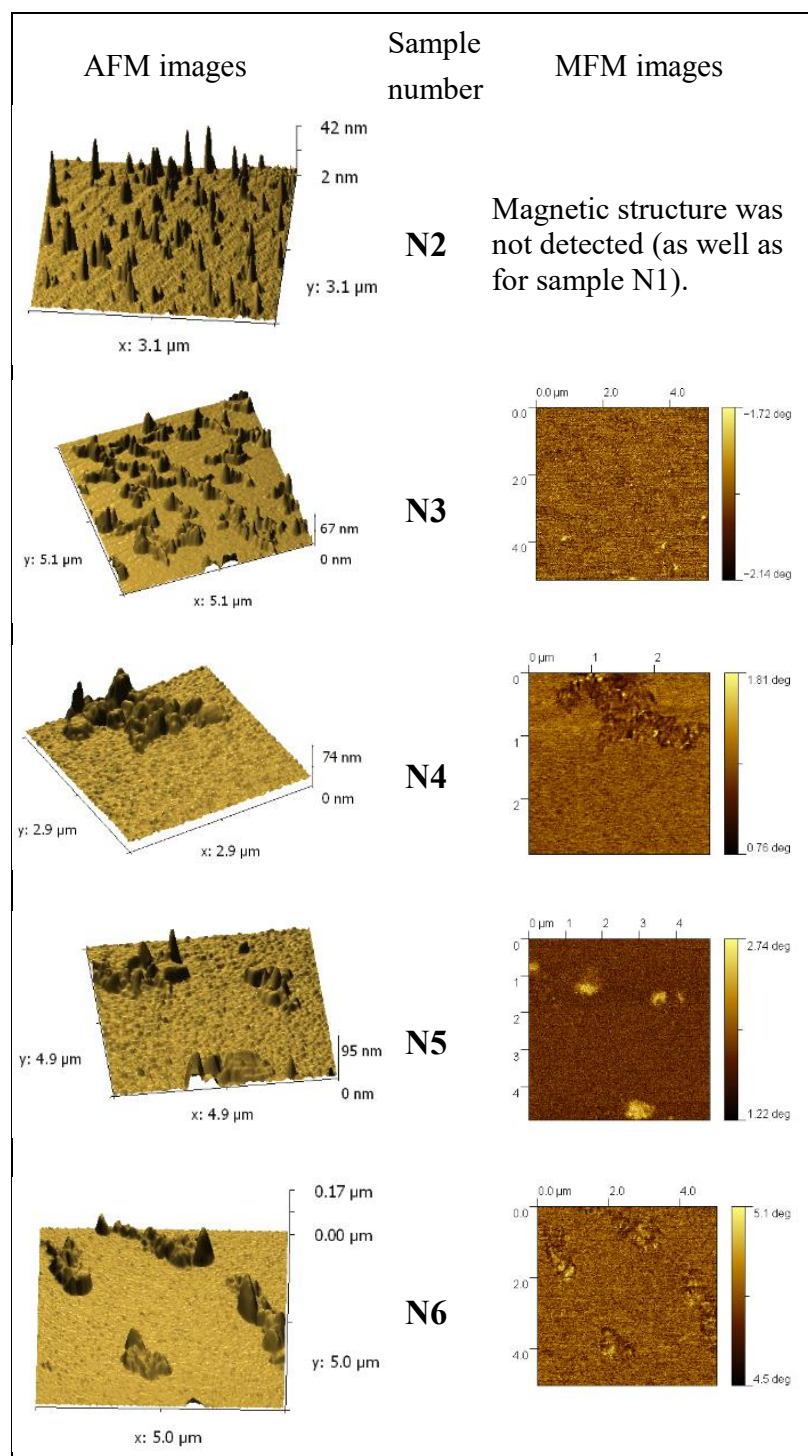

Fig. 1. Images for some InFeSb layers obtained with AFM (left) and MFM (right). (The images for sample N7 are similar those for sample N6.)

As the $\mathrm{Y}_{\mathrm{Fe}}$ parameter increases, these particles rise and start to form more and larger aggregates. Evidently, hot substrate promotes diffusion, which results in the formation of larger particles. The MFM images show that the surface formations are partially magnetic. A fine magnetic structure (light spots), which expands as the $T_{S}$ and $\mathrm{Y}_{\mathrm{Fe}}$ values increase, is observed on the surface particles. Apparently, the surface formations contain inclusions of iron, iron oxides and some FeSb variations.
Figure 2 depicts spectral dependences of the real, $<\varepsilon_{1}>(E)$, and imaginary, $<\varepsilon_{2}>(E)$, parts of the samples pseudo-dielectric function calculated using the ellipsometry data. Peaks corresponding to the optical transitions near the L critical point of the Brillouin zone in the InSb parent semiconductor are observed in the $\varepsilon_{2}(\mathrm{E})$ spectra of the InFeSb layers. It confirms that the crystal structure of the parent semiconductor is conserved in the layers under study.

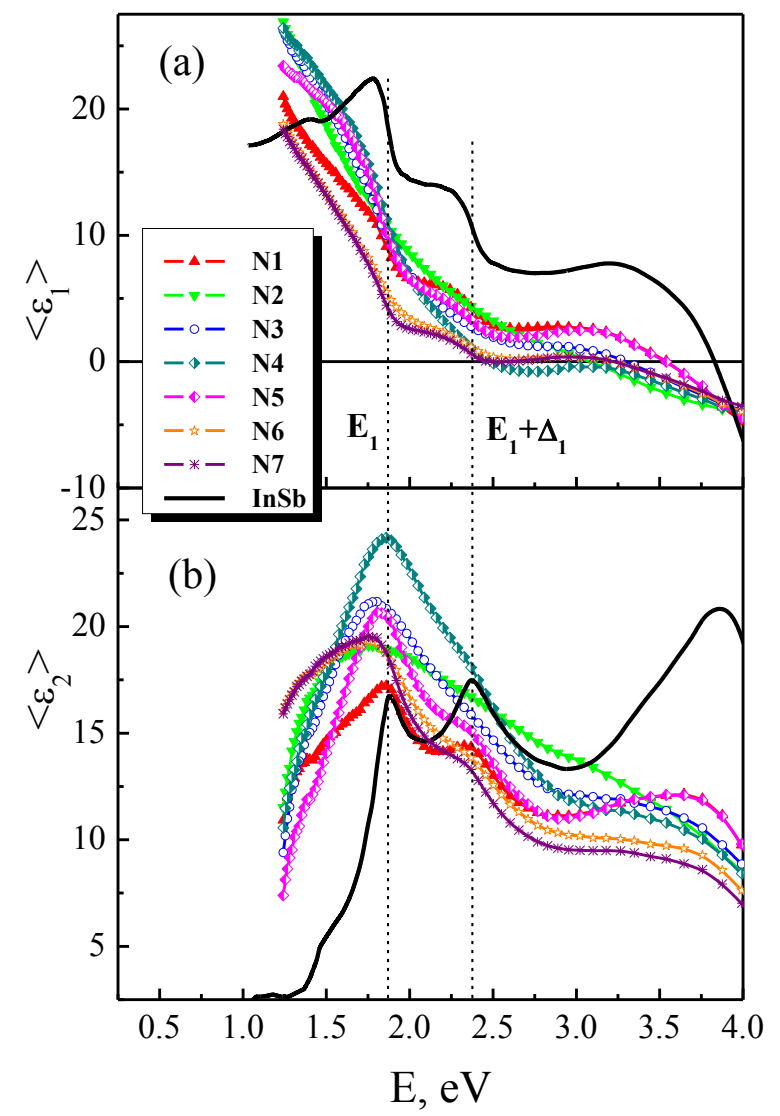

Fig. 2. Spectral dependences of the real, $<\varepsilon_{1}>$ (E), (a) and imaginary, $<\varepsilon_{2}>(E)$, (b) parts of pseudo-dielectric function for the investigated samples. Reported energies of the $E_{1}$ and $\mathrm{E}_{1}+\Delta_{1}$ transitions at the critical L point in InSb are shown by vertical dashed lines [3].

An increase of matrix imperfection with the Fe doping and the surface particles contribution are possible reasons for smearing the doublet $(\mathrm{E} \approx 1.85-2.36 \mathrm{eV})$ and increasing the maxima in the $\varepsilon_{2}(\mathrm{E})$ spectra as well as for rise in the $\varepsilon_{1}$ values in the region $\mathrm{E}<1.75 \mathrm{eV}$.

TKE room and low temperature spectra are displayed in Fig. 3(a,b). For the samples, whose the growth parameter is $\mathrm{Y}_{\mathrm{Fe}} \geq 0.12$, we observe large magnetooptical response even at room temperature. In the TKE spectra, two bands around 1.2 and $1.6 \mathrm{eV}$ are distinguished. Some peculiarities (kinks) are seen near $E_{1}$ and $E_{1}+\Delta_{1}$ transitions in the TKE spectra of the samples with high Fe content (N6 and N7). The TKE values significantly decrease when the $\mathrm{Fe}$ amount, $\mathrm{Y}_{\mathrm{Fe}}$, and/or growth temperature, $\mathrm{T}_{\mathrm{s}}$, gets smaller (samples N1 and N3). At room temperature the TKE signal from sample N2 is not detected. The weak TKE response of 
sample N5 is apparently associated with a lower density of the surface particles.

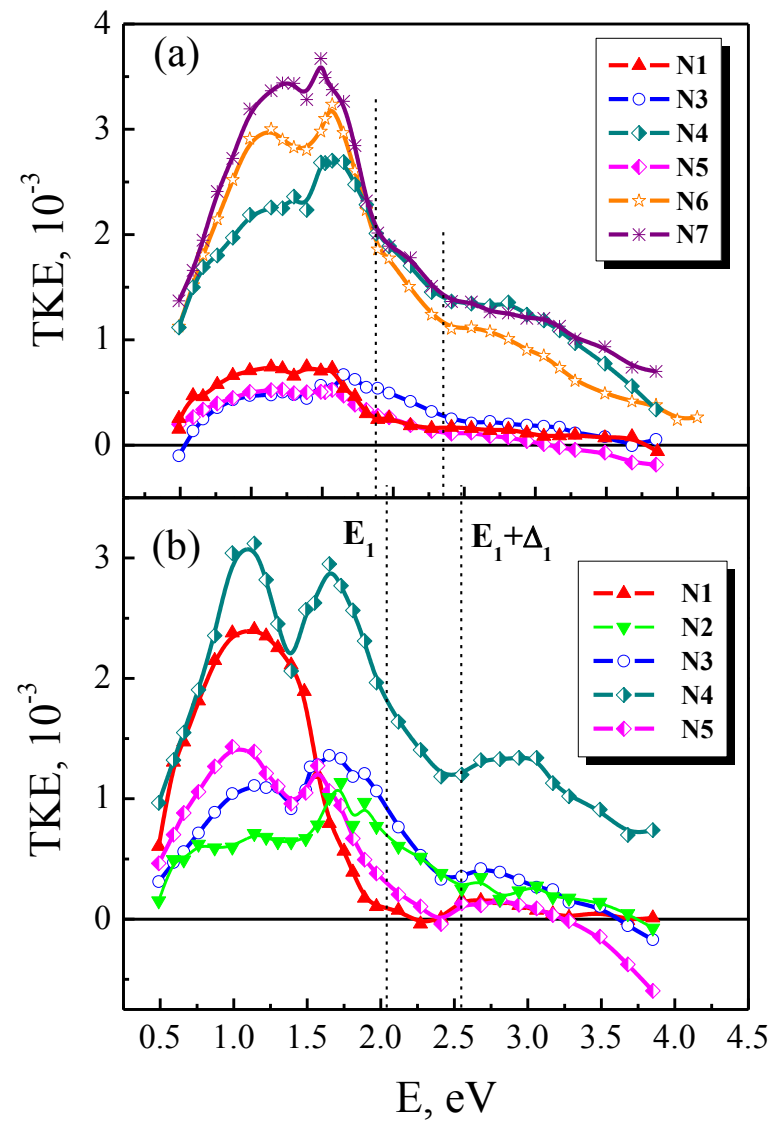

Fig. 3. Spectral dependences of TKE at room temperature (a) and at $\mathrm{T}=22 \mathrm{~K}(\mathrm{~b})$ for the investigated samples at $\mathrm{H}=2.5 \mathrm{kOe}$. Reported energies of the $E_{1}$ and $E_{1}+\Delta_{1}$ transitions at the critical $\mathrm{L}$ point in $\mathrm{InSb}$ are shown by vertical dashed lines for both room and low temperatures [3].

All the samples exhibit TKE signal increase as the temperature decreases. In the spectrum of sample N1 with the lowest Fe content even at the low temperature $\mathrm{T}=22 \mathrm{~K}$ only one band is present with the maximum around $1.2 \mathrm{eV}$. Similar band is observed in the spectra of other samples. There is a distinct peculiarity (minimum) around the $E_{1}+\Delta_{1}$ transition region in the low temperature TKE spectra and its location depends on the Fe content and growth temperature. We do not observe any peculiarities near the $\mathrm{E}_{1}$ transition, however, a strong band is present around $1.7 \mathrm{eV}$. Most likely, the TKE spectra represent superpositions of contributions from $\mathrm{InFeSb}$ ferromagnetic matrix and magnetic surface particles containing Fe oxides.

Studies of the temperature and field TKE dependences (Fig. 4) have shown that the InFeSb layers with $\mathrm{Y}_{\mathrm{Fe}}$ above 0.08 and $\mathrm{T}_{\mathrm{S}} \geq 200^{\circ} \mathrm{C}$ exhibit ferromagnetic behavior even at room temperature. At the same time, saturation in the fields used $(3 \mathrm{kOe})$ was observed only for sample N7 with the maximum $\mathrm{Fe}$ content. The TKE temperature dependences (Fig. 4a) display that the samples with the lower $\mathrm{Y}_{\mathrm{Fe}}$ values give more TKE contribution generated by a low-temperature ferromagnetic phase. It leads to the presence of well pronounced peculiarities in the TKE spectra related to this phase at temperatures lower than $100 \mathrm{~K}$. In the spectra of the samples prepared at bigger $\mathrm{Y}_{\mathrm{Fe}}$ values and $\mathrm{T}_{\mathrm{S}}$ higher than $200^{\circ} \mathrm{C}$, these peculiarities become less noticeable, since other magnetic phases make larger contribution to the effect.
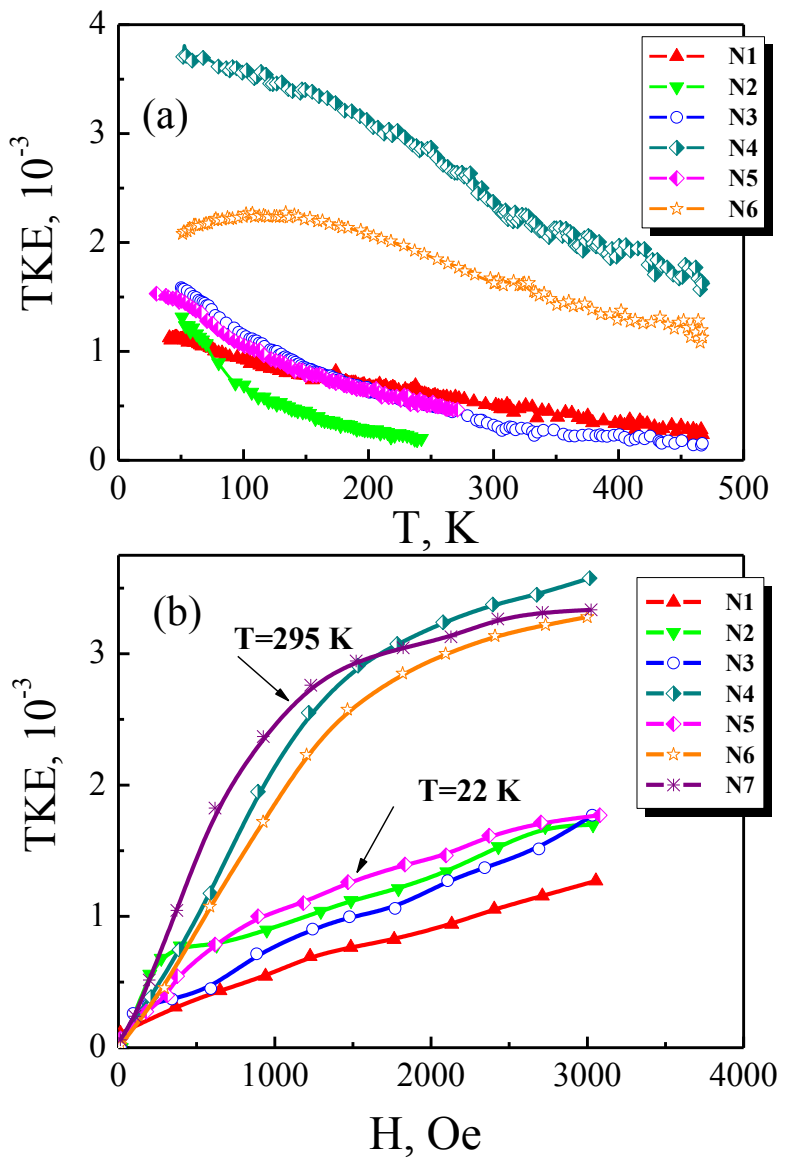

Fig. 4. (a) Temperature TKE dependences ( $E=1.73 \mathrm{eV}$ for $\mathrm{N} 1$, $\mathrm{N} 2$ and $\mathrm{N} 4 ; \mathrm{E}=1.65 \mathrm{eV}$ for $\mathrm{N} 3, \mathrm{~N} 5$ and $\mathrm{N} 6, \mathrm{H}=2.5 \mathrm{kOe}$ for all samples) and (b) field TKE dependences.

Figure 5 shows the normalized field TKE dependences, TKE $(\mathrm{H}) / \mathrm{TKE}\left(\mathrm{H}_{\max }\right) \sim \mathrm{M}(\mathrm{H}) / \mathrm{M}\left(\mathrm{H}_{\max }\right)$, for the samples with the lower $\mathrm{Fe}$ content and substrate temperatures (N1 and N2).

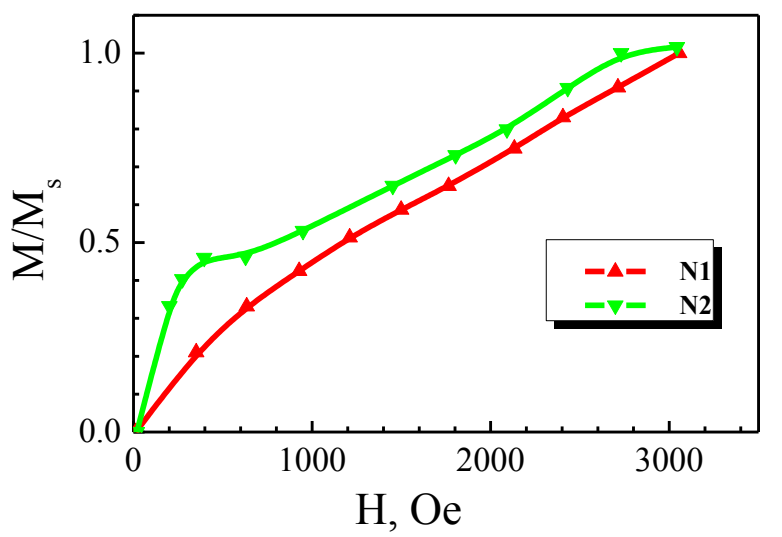

Fig. 5. Normalized field TKE dependences at $T=22 \mathrm{~K}$ for the samples with lower $\mathrm{Fe}$ concentrations and substrate temperatures. 
For sample N1, we observe approximately linear dependence on the magnetic field in the all temperature range. At the same time, a superposition of ferromagnetic and paramagnetic contributions is observed at low temperatures for sample N2.

\section{Conclusions}

Samples with $\mathrm{Y}_{\mathrm{Fe}}>0.08$ and $\mathrm{T}_{\mathrm{S}} \geq 200^{\circ} \mathrm{C}$ not only exhibit ferromagnetic behavior, but also have high Curie temperature. Even at $\mathrm{T}=194^{\circ} \mathrm{C}$ the $\mathrm{TKE}$ signal is also relatively strong. However, one should check, whether these properties are related to the bulk material or the surface particles, since the experimental data leads to a conclusion that these samples are multiphase. Most likely, the surface particles are compounds such as $\mathrm{FeO}$, $\mathrm{Fe}_{3} \mathrm{O}_{4}, \mathrm{Fe}_{2} \mathrm{O}_{3}$ (since the small particles being on the surface would inevitably oxidize), which are mixed with $\mathrm{FeSb}$ variations. Despite the fact that at room temperature a distinguishable signal is not detected from sample N2, it shows a low-temperature ferromagnetic response. However, without the substrate heating the overall effect is lower that may be related to less iron oxides content. Matrix doping in the sample with the Fe lowest content $\left(\mathrm{Y}_{\mathrm{Fe}}=0.08, \mathrm{~N} 1\right)$ is apparently insufficient to create ferromagnetism. Further improvement of the growth technology aimed at the fabrication of singlephase InFeSb layers or those with negligibly small content of undesirable phases, as well as the studies continuation are required to confirm the intrinsic hightemperature ferromagnetism of the $\mathrm{InFeSb}$ layers obtained by laser ablation.

This research was supported by the Russian Foundation for Basic Researches №15-02-02077

\section{References}

1. N. T. Tu, P. N. Hai, L. D. Anh, and M. Tanaka, Appl. Phys. Lett. 108, 192401 (2016).

2. A.V. Kudrin, Yu. A. Danilov et al. Trudy XI Mezhd. Symp. «Nanofizika i Nanoelectronika», v.1, 195 (2017).

3. V.I. Gavrilenko, A.M. Grekhov, D.V. Korbutyak, V.G. Litovchenko, Spravochnik "Optical properties of semiconductors", Kiev, Naukova Dumka, p.208 (1987). 